\title{
Quantum condensation from a tailored exciton population in a microcavity
}

\author{
P. R. Eastham ${ }^{1}$ and R. T. Phillips ${ }^{2}$ \\ ${ }^{1}$ Department of Physics, Imperial College London, London SW7 2BW, United Kingdom \\ ${ }^{2}$ Cavendish Laboratory, University of Cambridge, Cambridge CB3 OHE, United Kingdom
}

(Received 5 March 2009; published 7 April 2009)

\begin{abstract}
An experiment is proposed on the coherent quantum dynamics of a semiconductor microcavity containing quantum dots. Modeling the experiment using a generalized Dicke model, we show that a tailored excitation pulse can create an energy-dependent population of excitons, which subsequently evolves to a quantum condensate of excitons and photons. The population is created by a generalization of adiabatic rapid passage and then condenses due to a dynamical analog of the BCS instability.
\end{abstract}

DOI: 10.1103/PhysRevB.79.165303

PACS number(s): 71.36.+c, 03.75.Kk, 71.35.Lk, 78.67.Hc

\section{INTRODUCTION}

There is great interest in the possibility of quantumcondensed phases of solid-state quasiparticles, such as excitons, polaritons, and magnons. Such phases are characterized by the presence of a quantum state whose population scales with the size of the system and hence is much larger than one, i.e., macroscopic occupation. This is seen in recent experiments on Bose-Einstein condensation (BEC) of polaritons and polariton lasing. In these experiments, ${ }^{1-5}$ a semiconductor microcavity is excited at high energies, and a macroscopic population of low-energy polaritons emerges following relaxation and inelastic scattering. ${ }^{6}$ These condensates appear spontaneously, from states without macroscopic occupations. This differentiates them from optical parametric oscillator (OPO) experiments, ${ }^{7}$ where resonant pumping of polaritons leads directly to a macroscopic occupation.

The aim of this paper is to show how microcavities could be used to access condensation even in the absence of relaxation or inelastic scattering. We propose an experiment on a microcavity containing an ensemble of quantum dots, where the exciton decay times ${ }^{8}$ are $\sim 100$ ps. We demonstrate that the experiment could be faster than these decay times, so that energy relaxation and inelastic scattering would be negligible. Nonetheless, we shall show that a condensate develops. In contrast with a laser, this condensate is formed from part-matter part-light quasiparticles. In contrast with the OPO, it develops from a state with no macroscopic occupations, in the absence of the pump. And whereas relaxation is essential to obtain an equilibrium BEC or polariton laser, in our approach condensation occurs due to an instability of the Heisenberg dynamics. Our proposal implements, in the solid state, the type of dynamical condensation predicted in quenched Fermi gases. ${ }^{9}$

The first stage in our proposed experiment is the creation of a population of excitons in the quantum dots. We propose using a chirped laser pulse, which sweeps up through part of the inhomogeneously broadened exciton line. As shown by the demonstration of Rabi oscillations ${ }^{10}$ and density-matrix tomography, ${ }^{11}$ excitons in quantum dots are discrete twolevel systems, which can therefore be manipulated using laser pulses. The proposed pulse implements adiabatic rapid passage, which is a well-established technique for populating discrete states. ${ }^{12}$ It extends the technique, by controlling the pump spectrum to create an energy-dependent exciton population (Fig. 2).

The second stage occurs after the pump pulse has passed. It is the coherent quantum dynamics of the system, starting from the exciton population created by the pump. The pump is chosen such that this population is similar to a Fermi distribution with a sharp upper step. The system is described by a model similar to that which describes pair condensation in atomic gases and superconductors. A population with the form of a Fermi distribution could therefore condense due to a dynamical version of the BCS instability. ${ }^{9,13}$

The remainder of this paper is structured as follows. In Sec. II we outline our model for the dynamics of a microcavity containing quantum dots and explain how to identify a condensate in the solutions to this model. Section III presents numerical solutions to the model, showing both the pump and condensation stages. In Sec. IV we explain the condensation mechanism, and in Sec. V that of the pumping. In Sec. VI we discuss further the interpretation of our results as spontaneous condensation, some directions for future work, and highlight some other systems where similar condensation experiments could be considered. Finally, Sec. VII summarizes our conclusions.

\section{MODEL}

Our proposed experiment consists of a microcavity containing quantum dots, or more generally localized exciton states, pumped at a high angle by a chirped laser pulse. For simplicity, we suppose that the pump is circularly polarized and consider only one of the polarization states of the excitons. We model the quantum dots as a set of two-level systems, each describing the presence or absence of an exciton of the pump polarization in a given localized dot state. These localized excitons are coupled to the electromagnetic field by the dipole interaction. Since the exciton states in the dots are spatially separated, we neglect the non-radiative interactions between the different two-level systems. The appropriate Hamiltonian is then the generalized Dicke model. ${ }^{14}$

We consider a system of $N$ dots, and label the dots with an index $i$ so that $E_{i}$ is the energy of an exciton in the $i$ th dot. This state is localized at $\mathbf{r}_{i}$, with dipole-coupling energy $g_{i}^{0}$. We work with the collective couplings $g_{i}=\sqrt{N} g_{i}^{0}$, which are well defined in the large-system limit: $g_{i}$ becomes one-half of 
the Rabi splitting at resonance when all the dots have the same energy and coupling. The state of a dot is specified by the Bloch vector $\left\langle\boldsymbol{\sigma}_{i}\right\rangle$, where $\sigma_{i}^{-}=\sigma_{i}^{\mathrm{x}}-i \sigma_{i}^{\mathrm{y}}$ is the exciton annihilation operator and $\sigma_{i}^{z}$ is the inversion. The inversion is related to the occupation of the $\operatorname{dot} n_{i}$ by $n_{i}=\left(\sigma_{i}^{\mathrm{Z}}+1\right) / 2$ and is $-1(+1)$ for an unoccupied (occupied) dot.

Dicke models have previously been used to describe polariton condensation in equilibrium ${ }^{2,14-16}$ and in a dissipative open system. ${ }^{17}$ Here we are concerned with the opposite limit, of time scales short compared with the relaxation times. The dynamics therefore obeys the Heisenberg equation. Since we are concerned with condensation phenomena, involving large photon numbers, we treat the field classically. However, we retain the full quantum dynamics of the dots and hence the possibility of an incoherent population of excitons. In this approximation, the Heisenberg equation gives

$$
\begin{gathered}
i \dot{\psi}_{\mathbf{k}, t}=\omega_{\mathbf{k}} \psi_{\mathbf{k}, t}+\int g P_{\mathbf{k}, t}(E, g) d E d g+F_{\mathbf{k}, t}, \\
i \dot{P}_{\mathbf{k}, t}(E, g)=E P_{\mathbf{k}, t}(E, g)-g \sum_{\mathbf{k}^{\prime}} D_{\mathbf{k}-\mathbf{k}^{\prime}, t}(E, g) \psi_{\mathbf{k}^{\prime}, t}, \\
i \dot{D}_{\mathbf{k}, t}(E, g)=2 g \sum_{\mathbf{k}^{\prime}} P_{\mathbf{k}^{\prime}-\mathbf{k}, t}^{*}(E, g) \psi_{\mathbf{k}^{\prime}, t}-\text { c.c. },
\end{gathered}
$$

where $\psi_{\mathbf{k}}$ is the amplitude for an electromagnetic mode with in-plane wave vector $\mathbf{k}$ and energy $\omega_{\mathbf{k}}$, and $F_{\mathbf{k}}(t)$ is the (classical) pump. $D_{\mathbf{k}, t}(E, g)$ is a collective variable describing the inversion at wave vector $\mathbf{k}$,

$$
D_{\mathbf{k}}(E, g) \delta E \delta g=\frac{1}{A n} \sum_{i}\left\langle\sigma_{i}^{z}\right\rangle e^{-i \mathbf{k} \cdot \mathbf{r}_{i}},
$$

where the sum runs over states with $E \rightarrow E+\delta E$ and $g \rightarrow g$ $+\delta g$. The collective polarization, $P_{\mathbf{k}, t}$, is defined similarly, with $\sigma_{i}^{-}$replacing $\sigma_{i}^{z}$. Note that Eq. (1) generalizes the standard Maxwell-Bloch equations to allow for the distribution of $E_{i}$ and $g_{i}$. Thus the inversion and polarization become distributions over energy and coupling.

The fields $P_{\mathbf{k}, t}, \psi_{\mathbf{k}, t}$, and $F_{\mathbf{k}, t}$ have been normalized such that their square magnitudes are particle numbers per exciton state. A condensate is characterized by a macroscopic occupation number, i.e., one which scales with the size of the system. Thus an exciton-photon condensate has at least one $P_{\mathbf{k}, t} \sim N^{0}$ and one $\psi_{\mathbf{k}, t} \sim N^{0}$ (recall that $N$ is the total number of dots in the active region of the sample). In contrast, in a noncondensed state there is at most of order one particle per mode, and the $P_{\mathbf{k}, t}$ and $\psi_{\mathbf{k}, t}$ are all $\lesssim N^{-1 / 2}$.

The approximation leading to Eq. (1) is the standard semiclassical approximation, used to treat condensates including lasers, ${ }^{18}$ superconductors, BCS superfluids, ${ }^{9}$ and polariton condensates. ${ }^{19}$ It neglects the quantum fluctuations of the electromagnetic field, which dominate if the photon number is small, i.e., close to threshold with a small number of dots. ${ }^{18,19}$ Since we could have $N \sim 10^{5}$, the semiclassical approximation is in general very well controlled. However, as in all semiclassical treatments of condensation, we must supplement Eq. (1) with noise terms to obtain a correct de- scription of the dynamics. Without such terms the noncondensed solution remains a steady-state above threshold. However, it becomes unstable and hence is not realized. We therefore add a perturbation driving the cavity field in Eq. (1), modeling, for example, spontaneous emission into the cavity modes. The form and strength of this perturbation does not affect our results: we show results with Gaussian white noise but have obtained similar results using a deltalike kick.

We now specialize Eq. (1) to develop a simulation of our proposed experiment. We introduce imaginary parts to $\omega_{\mathbf{k}}$ to allow for the decay of the microcavity photons, with timescales of a few picoseconds. ${ }^{1}$ The initial condition is $\left\langle\sigma_{i}^{z}\right\rangle=$ -1 , and there are many exciton states distributed over the active area of the sample. Thus the sum in Eq. (2) is strongly peaked near $\mathbf{k}=0$, and we approximate the initial conditions as $D_{\mathbf{k}, t}(E, g)=\delta_{\mathbf{k}} D_{0, t}(E, g)$. The dynamics is then that of a continuous medium due to motional narrowing, with the short-range spatial structure of the exciton states averaged out on the long scales of the photons.

We consider a plane-wave pump, at a high angle where the excitons lie outside the stopbands of the mirrors. The field acting on the dots at this wave vector, $\mathbf{k}_{p}$, may then be taken as the driving field. Anticipating our analysis of the condensation, we retain only one other mode of the field, specifically the confined cavity mode with $\mathbf{k}=0$. This reduces Eq. (1) to

$$
\begin{gathered}
i \dot{\psi}_{0}=\omega_{0} \psi_{0}+\int g P_{0}(E, g) d E d g \\
i \dot{P}_{0}(E, g)=E P_{0}(E, g)-g D_{0}(E, g) \psi_{0}, \\
i \dot{P}_{p}(E, g)=E P_{p}(E, g)-g D_{0}(E, g) F_{p}, \\
i \dot{D}_{0}(E, g)=2 g\left(P_{p}^{*}(E, g) F_{p}+P_{0}^{*}(E, g) \psi_{0}\right)-\text { c.c. },
\end{gathered}
$$

corresponding to an ensemble of two-level systems, interacting with two modes of the field. $P_{p}$ is the polarization at the pump wave vector and $F_{p}$ the driving field.

\section{NUMERICAL RESULTS}

We have simulated our proposed experiment by solving Eq. (3) numerically with $N=4500$ two-level systems. Results are shown in Figs. 1 and 2. These results focus on a model with a single coupling strength $g$ and a Gaussian distribution of exciton energies with variance $\sigma^{2}$. We take the pump to be a linearly chirped Gaussian,

$$
2 g F_{p}(t)=\frac{S}{\sqrt{2 \pi \tau^{2}}} e^{-i\left(\nu_{0}+\alpha t / 2\right) t} e^{-t^{2} /\left(2 \tau^{2}\right)},
$$

where $S=\int 2 g\left|F_{p}(t)\right| d t$ is the usual pulse area. The pulse time $\tau$ defines dimensionless times and energies, and the zero of energy is chosen at the center of the exciton line. The remaining parameters are $\sigma=15 \hbar / \tau, \mathfrak{R}\left(\omega_{0}\right)=-5 \hbar / \tau, \quad \nu_{0}=$ $-20 \hbar / \tau, \quad \alpha=5 / \tau^{2}, \quad-\Im\left(\omega_{0}\right)=1.5 \hbar / \tau, g=13 \hbar / \tau$ and $S=5 \pi$. 


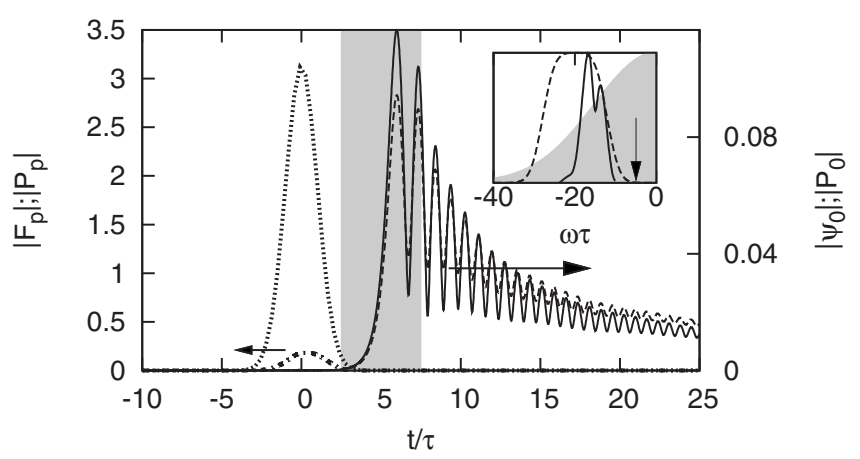

FIG. 1. Electromagnetic fields and polarizations as functions of time for the simulation described in the text. Dotted: pump field $\left|F_{p}\right|$ (left axis). Dot-dashed: polarization $\left|P_{p}\right|$ at pump wavevector, integrated over dot energies and couplings (left axis). Solid: cavity field $\left|\psi_{0}\right|$ at $\mathbf{k}=0$ (right axis). Dashed: polarization $\left|P_{0}\right|$ at $\mathbf{k}=0$ (right axis). Inset: spectrum $\left|\psi_{0}(\omega)\right|^{2}$ during the shaded region of the main plot (solid curve). The pumped population (dashed curve), exciton energy distribution (shading), and energy of the $\mathbf{k}=0$ cavity mode (arrow) are shown for comparison.

These parameters, with $\tau=3 \mathrm{ps}$, are reasonable for a microcavity containing interfacial quantum dots. Although there is a distribution of $g$ due to the different sizes of the dot states, this does not qualitatively change our results.

Figures 1 and 2 are the key results of this paper, demonstrating the scenario outlined in Sec. I. Referring first to Fig. 1 , we see that there can indeed be two stages to the dynamics. In the first stage, during the pump pulse, $P_{0}$ and $\psi_{0}$ are vanishingly small. $P_{p}$ does become finite, reflecting the fact that the pump laser does induce some coherent polarization in the excitons. However, this induced condensate has disappeared, $P_{p} \ll 1 / \sqrt{N}$, by the end of the pumping stage. We therefore argue that the pump produces an incoherent population of excitons. Furthermore, as shown in Fig. 2, this population has a sharp upper step, similar to that of the Fermi function at a low effective temperature $T \sim 1 / \tau$.

In the second stage, visible in Fig. 1, we see both $\psi_{0}$ and $P_{0}$ building up to values of order $N^{0}$. This directly demonstrates condensation of excitons and photons.

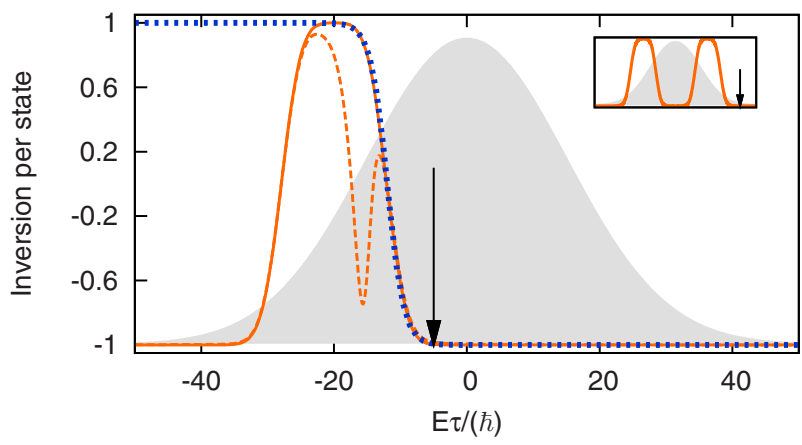

FIG. 2. (Color online) Solid: Simulated exciton inversion profile immediately after pumping $(t / \tau=3)$ showing the population created by the pump pulse [Eq. (4)] and by a superposition of such pulses (inset). Dotted: equilibrium exciton distribution with fitted temperature $\hbar /(4.2 k \tau)$; this is $0.6 \mathrm{~K}$ for $\tau=3$ ps. Arrows: energy of the $\mathbf{k}$ $=0$ cavity mode. Dashed: exciton population during the condensation $(t / \tau=7)$.

\section{CONDENSATION MECHANISM}

To understand the condensation, we consider the dynamics of the pumped population. Linearizing Eq. (1) gives normal modes which are plane waves, with $\psi_{\mathbf{k}, t}=e^{-i \lambda_{\mathbf{k}} t} A_{\psi}$ and $P_{\mathbf{k}, t}=e^{-i \lambda_{\mathbf{k}} t} A_{P}(E, g)$. Their frequencies $\lambda_{\mathbf{k}}=\lambda_{\mathbf{k}}^{\prime}+i \lambda_{\mathbf{k}}^{\prime \prime}$ obey $^{13}$

$$
\omega_{\mathbf{k}}-\lambda_{\mathbf{k}}=-\int \frac{\nu(E)}{E-\lambda_{\mathbf{k}}} d E,
$$

where $\nu(E)=\int g^{2} D_{0}(E, g) d g$ is an optical density of the excitons. Applying this result to the state immediately after the pump pulse, we find an unstable mode at $\mathbf{k}=0$. This instability gives the exponential growth of the polarization and field to macroscopic values visible in Fig. 1. In fact, Eq. (5) predicts instabilities for $|\mathbf{k}|<k_{c}$. The fastest-growing mode is at $\mathbf{k}=0$, and as it grows, it suppresses the gain for the others. It will thus be dynamically selected, and we therefore neglected cavity modes with $\mathbf{k} \neq 0$ in the simulations.

For the parameters used here, the instability predicted by Eq. (5) corresponds to the BCS instability in a superconductor. This can be seen by considering Eq. (5) close to an instability, where it becomes $\left(g_{i}=g\right)$ :

$$
\begin{gathered}
\frac{\omega_{\mathbf{k}}^{\prime}-\lambda_{\mathbf{k}}^{\prime}}{g^{2}}=-\mathcal{P} \int \frac{D_{0}(E)}{E-\lambda_{\mathbf{k}}^{\prime}} d E, \\
\lambda_{\mathbf{k}}^{\prime \prime}=\pi g^{2} \operatorname{sgn}\left(\lambda_{\mathbf{k}}^{\prime \prime}\right) D_{0}\left(\lambda_{\mathbf{k}}^{\prime}\right)-\gamma .
\end{gathered}
$$

Equation (6b) describes the growth or decay of the normal mode, with the first term as the gain/loss from the excitons and the second term as the loss due to the cavity decay $\gamma=$ $-\Im\left(\omega_{\mathbf{k}}\right)$. Equation (6a), which determines the energy of the mode, is the Cooper equation of the BCS model. ${ }^{20}$ The term corresponding to the usual pairing interaction is $g^{2} /\left(\lambda_{\mathbf{k}}\right.$ $\left.-\omega_{\mathbf{k}}\right)$, which we recognize as the effective interaction between excitons, mediated by the cavity modes. Here it is an attractive interaction, as required for BCS, since the excitons lie below the photons. The term corresponding to the Fermi distribution is the exciton population created by the pump. In a superconductor, there is a solution to the Cooper equation below the Fermi energy due to the step in the Fermi distribution; in the same way, Eq. (6a) has a solution below the step in the exciton occupation. Equation (6b) shows that this mode experiences gain and hence can become unstable.

To confirm the origin of the condensation, we show in the inset to Fig. 1 the spectrum of the $\mathbf{k}=0$ field and in Fig. 2 the population during condensation. As expected, the condensate is at a frequency below the step in the exciton population (Fig. 1); this leads to hole burning there (Fig. 2). Reducing the center frequency of the chirp, $\nu_{0}$, we find that the condensation stage in Fig. 1 disappears as the effective interaction decreases below its critical value. The long-time limit of the simulations is then just the population of excitons.

Since our condensate occurs on timescales short compared with the relaxation times, it will not be in equilibrium. This leads to phenomena absent for an equilibrium condensate. In Fig. 1, for example, we see a slow decay of the condensate, with the ringing oscillations predicted for atomic gases. ${ }^{9,13,21}$ 


\section{PUMP MECHANISM}

It is perhaps surprising that our pump configuration could generate an incoherent population, without macroscopic occupation. To explain the mechanism, we note first that the driving field dominates during the pump stage (Fig. 1). Thus the pumping can be understood in terms of the well-known dynamics of noninteracting two-level systems driven by a chirped laser pulse. ${ }^{12}$ After eliminating the time-dependent frequency $\omega(t)$ of the pump with a unitary transformation, this dynamics is a precession of the Bloch vectors $\left\langle\boldsymbol{\sigma}_{i}\right\rangle$ around axes $\mathbf{B}_{i}=\left[2 g_{i} R(t), 0, \omega(t)-E_{i}\right]$, where $R(t)$ is the pump field at the dot.

Consider first a dot with energy inside the chirp. Provided the pump is strong enough, the Bloch vector of such a dot adiabatically follows $\mathbf{B}_{i}$ from $-\hat{\mathbf{k}}$ to $\hat{\mathbf{k}}$, and it becomes populated. Furthermore, a dot with energy outside the range of the chirp will not respond unless the pump is very strong, and hence such a dot will remain unpopulated. Thus we see that the only dots which could be polarized by the pump are those at the edge of the chirp. However, there are very few such dots. Furthermore, any polarizations induced in dots with different energies would have different phases due to the chirping. Thus, as shown in Fig. 1, the chirped pump does not induce a collective polarization. Noting additionally that there are no cavity modes resonant with the pump, we see why the pump generates a state with $P_{\mathbf{k}, t}, \psi_{\mathbf{k}, t} \ll N^{-1 / 2}$.

\section{DISCUSSION}

In common with previous experiments on polariton condensation, polariton lasing, and the OPO, our proposal involves excitation of a microcavity with a laser. This has apparently created controversy for the previous experiments because a defining characteristic of a condensate is macroscopic coherence. Since the system is being driven with a coherent field, it might be argued that the condensation is induced by the pumping. This would clearly be the case with resonant excitation of bosonic modes, such as the polaritons: the linear coupling between the polariton modes and the laser will ensure a large occupation of the polaritons over a small frequency range. However, the situation is different for the chirped pumping of few-level systems we are proposing. As we have explicitly shown, this does not directly create a state with a large occupation. This is not quite sufficient to prove that the condensation is spontaneous since in an ensemble of few-level systems there might be echo phenomena, where the pump coherence re-emerges after a delay. This is unlikely to be the explanation for our simulation results since they agree with the predictions of Eq. (5). The theory could similarly be used to rule out alternative explanations of a real experiment.

The possibility that the condensation is an echo of the pump can be more explicitly excluded by examining whether the phase of the condensate depends on the phase of the pump. We have done this in our simulations by examining the phase spectrum of the condensate (associated with the power spectrum in the inset to Fig. 1). We find that this phase spectrum is the same in simulations with different pump phases but the same random noise. Thus the condensate phase is not related to the phase of the pump but to that of the noise, which provides the perturbation required to break the gauge symmetry. That the condensate phase comes from a perturbation is standard for spontaneous symmetry breaking. ${ }^{22}$ In particular, it also occurs in semiclassical laser theory, ${ }^{18}$ where the classical field is created by amplification of spontaneous emission noise.

A fully quantitative description of our proposed experiment is likely to require a more detailed model of the quantum dot states. It would also be desirable to extend our model to (a) incorporate relaxation processes, which on longer timescales will drive the off-equilibrium condensate toward an equilibrium one, ${ }^{13}$ and (b) develop a full quantum theory, as has been done for photon lasers, ${ }^{18}$ polariton lasers, $, 5,23,24$ and equilibrium condensates. ${ }^{19}$ Finally, it would be interesting to compare this work with Ref. 25, which appears to show a type of dynamical condensation, under very different conditions.

Although the parameters used here have been chosen to suit interfacial quantum dots, ${ }^{8,26}$ many other choices are possible. The basic requirements are that the pumping is slow enough to create a controlled inversion profile within the inhomogeneous line, such that the couplings can be increased so that the instability occurs after the pump pulse but before the excitons decay. Other systems described by generalized Dicke models, such as Fermi gases, StranskiKrastanow dots in microcavities, or Josephson junction arrays, could be considered.

\section{CONCLUSIONS}

To conclude, we have proposed and analyzed an approach to quantum condensation in a solid-state system. The key is the chirped pumping [Eq. (4)], which we have shown can create an energy-dependent population in the exciton line. Such a population can condense even in the absence of relaxation or inelastic scattering. Since our approach uses the spectrum of the pump to tailor the population, it would be possible to pump other initial states (inset to Fig. 2). Thus our technique could be used more generally, to explore the quantum dynamics of a many-particle system from controlled initial conditions.

\section{ACKNOWLEDGMENT}

This work was supported by EPSRC Grants No. EP/ F040075/1 and No. EP/C546814/01. 
${ }^{1}$ H. Deng, D. Press, S. Gotzinger, G. S. Solomon, R. Hey, K. H. Ploog, and Y. Yamamoto, Phys. Rev. Lett. 97, 146402 (2006).

${ }^{2}$ J. Kasprzak et al., Nature (London) 443, 409 (2006).

${ }^{3}$ R. Balili, V. Hartwell, D. Snoke, L. Pfeiffer, and K. West, Science 316, 1007 (2007).

${ }^{4}$ D. Bajoni, P. Senellart, E. Wertz, I. Sagnes, A. Miard, A. Lemaitre, and J. Bloch, Phys. Rev. Lett. 100, 047401 (2008).

${ }^{5}$ A. P. D. Love et al., Phys. Rev. Lett. 101, 067404 (2008).

${ }^{6}$ T. D. Doan, H. T. Cao, D. B. Tran Thoai, and H. Haug, Phys. Rev. B 74, 115316 (2006).

${ }^{7}$ R. M. Stevenson, V. N. Astratov, M. S. Skolnick, D. M. Whittaker, M. Emam-Ismail, A. I. Tartakovskii, P. G. Savvidis, J. J. Baumberg, and J. S. Roberts, Phys. Rev. Lett. 85, 3680 (2000).

${ }^{8}$ W. Langbein and B. Patton, Phys. Rev. Lett. 95, 017403 (2005).

${ }^{9}$ A. V. Andreev, V. Gurarie, and L. Radzihovsky, Phys. Rev. Lett. 93, 130402 (2004); R. A. Barankov and L. S. Levitov, ibid. 93, 130403 (2004); E. A. Yuzbashyan, O. Tsyplyatyev, and B. L. Altshuler, ibid. 96, 097005 (2006).

${ }^{10}$ A. J. Ramsay et al., Phys. Rev. B 75, 113302 (2007).

${ }^{11}$ Y. Wu, X. Li, L. M. Duan, D. G. Steel, and D. Gammon, Phys. Rev. Lett. 96, 087402 (2006).

${ }^{12}$ V. S. Malinovsky and J. L. Krause, Eur. Phys. J. D 14, 147 (2001).

${ }^{13}$ P. R. Eastham, J. Phys.: Condens. Matter 19, 295210 (2007).

${ }^{14}$ J. Keeling, P. R. Eastham, M. H. Szymanska, and P. B. Littlewood, Phys. Rev. B 72, 115320 (2005).
${ }^{15}$ P. R. Eastham and P. B. Littlewood, Phys. Rev. B 64, 235101 (2001).

${ }^{16}$ P. R. Eastham and P. B. Littlewood, Solid State Commun. 116, 357 (2000).

${ }^{17}$ M. H. Szymanska, J. Keeling, and P. B. Littlewood, Phys. Rev. B 75, 195331 (2007).

${ }^{18}$ H. Risken, in Progress in Optics, edited by E. Wolf (NorthHolland, Amsterdam, 1970), Vol. 8, Chap. 5, pp. 241-294.

${ }^{19}$ P. R. Eastham and P. B. Littlewood, Phys. Rev. B 73, 085306 (2006).

${ }^{20}$ P. G. de Gennes, Superconductivity of Metals and Alloys (Westview, Boulder, CO, 1999).

${ }^{21}$ The oscillations are not related to those predicted for the OPO in I. A. Shelykh, M. M. Glazov, D. D. Solnyshkov, N. G. Galkin, A. V. Kavokin, and G. Malpuech, Phys. Status Solidi C 2, 768 (2005).

${ }^{22}$ J. W. Negele and H. Orland, Quantum Many-Particle Systems (Addison-Wesley, Redwood City, CA, 1988).

${ }^{23}$ F. P. Laussy, G. Malpuech, A. Kavokin, and P. Bigenwald, Phys. Rev. Lett. 93, 016402 (2004).

${ }^{24}$ D. M. Whittaker and P. R. Eastham, arXiv:0811.4333 (unpublished).

${ }^{25}$ H. Vinck-Posada, B. A. Rodriguez, P. S. S. Guimaraes, A. Cabo, and A. Gonzalez, Phys. Rev. Lett. 98, 167405 (2007).

${ }^{26}$ A. G. Steffan and R. T. Phillips, Phys. Status Solidi A 190, 541 (2002). 